\title{
L'ESPONSALICI DINS LA PRĀCTICA MATRIMONIAL CATALANA AL S. XII
}

\author{
Maria Pont
}

I que va prendre en dot dos rossos

lots de cavalls,

un de cabres,

un sac d'or,

un flascó en forma de conquilla,

un rentamans (ple) de neu, un tupí amb grans de mill,

una sitga de deu colzes plena de cebes

i una enormitat de porcelles.

Diuen que d'aquesta manera Cotis de Tràcia

va casar la filla amb Ifícrate.

(ANAXANDRIDES, Protesilao. (Atenea, Deipnosoph, IV, 131)).

*De la mateixa manera, Firch, quan considera les ocasions en ies quals es produeixen els intercanvis cerimonials a la Polinèsia, enumera naixements, iniciacions, matrimonios, malalties, morts i encara altres incidents de la vida social o aspectes del rituals. ( $R$. FIRTH, Primitive Polinesian Economics. Londres 1939, P. 321 en C. Lévi-Strauss: Les Estructures Élémentaires de la Parenté. ParisMouton. 1968, p. 61).

No hem de perdre de vista que existeixen pràctiques i creences especials de certs pobles $\mathrm{i}$ de certes civilitzacions. Aquestes pràctiques i creences tenen sempre la tonalitat particular que vesteix cada fenomen en cada societat. Per molt indefinits que siguin els límits de la seva extensió, corresponen a fets d'estructura que expressen corrents de civilització. Per això la sociologia no pot fer-se 
al marge de l'etnografia i de la història ${ }^{1}$. I per això la història no pot consolidar-se al marge de l'etnografia $\mathrm{i}$ de la sociologia. Aquesta interdisciplinaritat fa que el camí a seguir en la investigació de qualsevol fet històric prengui d'altres perspecrives. És la in tenció d'aquest treball.

Si mirem les definicions del mot esponsalici com a fenomen aillat d'una realitat social, trobarem explicacions com ara erelatiu o pertanyent a les esposalles», o «acte previ al matrimoni consistent en el compromís mutu de casaments, o sacte solemne fet entre els dos promesos i llurs famílies amb la participació de parents i amics i sovint amb la presència del sacerdot o del cap religiós i que comportava l'intercanvi dels dons esposalicis». D'aquestes tres explicacions, en podem, extreure una síntesi: l'esponsalici s'explica com un fet de dret ${ }^{2}$, com una execució legal d'una realitat social, les esposalles, és a dir, la legitimació d'una pròxima unió, pel vin. cle del matrimoni, entre un home i una dona amb el consentiment de llurs famílies. Això fora tot. Ara bé, si volem anar més lluny en el camp de la recerca, també cal anar més lluny en el camp de les preguntes. ¿Podem explicar l'esponsalici com un fet social d'intercanvi, de donacions recípróques entre els futurs contraents? $?^{3}$. El que M. Mauss anomenaria un afet social totals? ${ }^{4}$. De fet, l'esponsalici és un acte previ a. un compromís - escrit o oral - entre dues parts amb l'obligació de... contreure matrimoni. En resum, la noció de cerimònia i de compromís que legitimarà l'acte previ al matrimoni crearà l'obligació - legal o moral- de dur-lo a terme. Però cercant la via antropològica en el sentit del discurs, l'intercanvi dels béns esponsalicis, pedra de toc del con-

1 M. MaUSs, Introducción al anälisis de los fenómenos religiosos, 1908.

2 Pertanyent al dret privat català, d'origen romà.

3 «auss s'est proposé de montrer, d'abord que l'échange se présente, dans les sociétés primitives, moins sous forme de transactions que de dons réciproques; ensuite, que ces dons réciproques occupent une place beaucoup plus importante dans ces sociétés que dans la nôtre». (Cl. LEvvl.STRAuss: Les Estructures Elementaires de la Parenté, Paris-Mouton, 1968, p. 61).

4 s...enfin, que cette forme primitive des échanges n'a pas seulement, et n'a pas essentiellement, un caractère économique mais nous met en présence de ce qu'il appelle (Mauss) heureusement "un fait social total", c'est-à-dire doué d'une signification à la fois sociale et religieuse, magique et économique, utilitaire et sentimentale, juridique et morales. (Cl. LEVI-STrAUSS, op. cit. p. 61). 
tracte, ens donarà llum per a una interpretació més profunda del fet.

Ens cal centrar-nos en el temps que ens ocupa aquest treball: 1143-1193. Catalunya i un recull de documentació esponsalícia d'aquest períodes. El que primer apareix, en una prèvia lectura d'un document esponsalici, és el seu caràcter de legalitat, de contracte ordenat. També ens apareix, o almenys la intuïm per l'essència del contracte, la seva indissolubilitat ${ }^{6}$, fruit de la moral de l'època. Estem, i cal no oblidar-ho, en el s. XII. L'Església era l'única que podia legitimar una futura unió matrimonial, un acte previ al matrimoni. A primera vista, doncs, ens és facil de detectar un esponsalici, la paraula clau hi és: sponsalicio, in sponsalicium. Continuem interrogant el document a nivell formal. En alguns casos el subjecte actiu del document, és a dir qui el fa, és el futur espòs; en d'altres casos és la dona, però n'hi ha pocs. La fórmula, ometent els noms, és la següent: Ego...dono tibi... in sponsalicio... i un seguit de béns, cases, terres, diners, etc. pertanyents a la persona que fa el document, és a dir al futur marit o al marit, cosa que també és fâcil de detectar; depèn si anomena la dona, en un temps futur - aqui spero babere per coniugew-, o en un temps present - «dono tibi uxore mee»-7.

Aquest marc formal del document que tenim a les mans ens fa veure, doncs, un compromís, dues persones, un patrimoni i un intercanvi. Un intercanvi? M'explicaré. Les dades, i continuo encara en una primera lectura del document, dels béns materials que es cedeixen a l'altra part, hi són. Dades que seran vàlides per a poder catalogar el patrimoni del marit. Un intercanvi? Estem en el segle XII. Les dones difícilment tenien un patrimoni fort, si no pertanyien a un estament social poderós. La dona, la futura esposa

5 Els esponsalicis no són abundants. De fet el contracte per escrit d'una futura unió matrimonial estava limitat a estaments acomodats d'aquesta època. Els esponsalicis estudiats pertanyen al fons de Cancelleria de l'A C. A. de Barcelona.

6 Ev. S. MATEU, 1, 18-21: el fou així com Jesucrist va tenir al món: Maria, la seva mare, estant promesa amb Josep, abans de viure junts, es trobà que havia concebut de l'Esperit Sants. Nou Testament, Ed. Monjos de Montserrat (1961) (nota: El contracte de prometatge entre els jueus no es podia desfer sense un repudi legal).

7 Cal tenir en compte que aquesta donació de béns esponsalicis es pot fer abans o després del matrimoni, donació esponsalicia en el primer cas i escreix -augment que fa el marit de la dot de la dona - en el segon. 
- l'esposa, aportarà al matrimoni, en la majoria dels casos, el seu aixovar i prou. ¿I el seu aixovar por intercanviar-se per cases, terres i patrimoni? No. La dona aportava quelcom més important en aquella època: la seva virginitat i la seva maternitat. Ella i només ella podia donar la continuittat al llinatge del seu marit, portant al món, després del matrimoni, el fill baró. Un intercanvi, doncs.

I la paraula intercanvi ens posa en el camí de l'antropologia ${ }^{8}$. I la mateixa paraula porta immers el contingut de reciprocitat. Serà l'inter-canvi per la via del do?. Donar i rebre, dues paraules clau dins de l'intercanvi i, en el nostre cas, dins de l'intercanvi dels béns esponsalicis. Dintre de tota aquesta teoria de l'intercanvi del do i del contrado, posada a la llum per M. Mauss i anomenada pel mateix autor un «sistema de prestacions totals», hi ha una vessant anomenada potlach, que consisteix en un sistema de prestacions - dons - i contraprestacions - contradons- de fals matís voluntari, ja que l'obligatorietat que així es compleixi és patent en el seu mateix contingut ${ }^{10}$. L'obligació essencial del potlach és donar i rebre. Aquest mecanisme de redistribució tindrà, entre altres, dues funcions importants: en primer lloc un valor moral de qui comença el potlach, que podem denominar de prestigi. ¿No pot equivaler a l'home que comença a posar a la llum els seus béns pa. trimonials, o millor dit els seus béns esponsalicis en un paper? I en segon lloc, l'obligació de retornar-ho, de la manera que sigui, per part de qui ho ha rebut. ¿No queda en deute la seva futura o legal muller? El potlach també solidifica l'aliança entre les dues bandes que s'intercanvien dons. ¿No és important, al s. XII i a Catalunya, el sentit d'aliança entre els dos llinatges d'on han sortit els esposos? Tot això ve determinat per una festa. L'esponsalici no pot ser aquesta festa? El mateix Mauss, en estudis posteriors ${ }^{11}$, estableix la praxis de la seva teoria. L'autor diu: «El potlach forma

${ }^{8}$ Per aquesta questió, M. Msuss: Ensayo sobre el don, en Sociología y Antropologia, Ed. Tecnos, Madrid, 1971.

9 Op. cit. M. Mauss: Ensayo sobre el don.

10 El potlach és un costum caracteristic de les Illes del Pacific i de la costa del N.O. del Canadà i Alaska. Mauss es va basar per a l'estudi d'aquest fenomen de distribució de riquesses en els estudis etnogràfics de Boas i Malinowski (CI. LFVI. STRAUSS: op. cit., p. 61).

${ }^{11}$ M. MAUss: Sociedad y Ciencias Sociales. Don, contradon y intercambio. LLa extensión del potlach en Melanesiav [1920] (Barral Editores, Barcelona, 1970, pp. 27-32). 
part d'un sistema que nosaltres hem proposat de denominat "sistema de prestacions totals" que és un sistema normal en totes les societats basades en clans. Ja que l'exogàmia és un intercanvi de totes les dones dels clans lligats per llaços cognàtics» ${ }^{12}$. ¿No és aquesta la nostra societat medieval, unida per forts sistemes d'aliança i de filiació mitjançant sòlides estructures de parentiu? No configura el llinatge el sistema de filiació, per matrimoni entre cosins encreuats en alguns $\operatorname{casos}^{13}$, determinant d'aquest periode? Són moltes les similituds de la cerimònia esponsalícia amb el po. tlach melanesi. Potser també cal veure d'altres trets del potlach en altres civilitzacions no tant «primitives». Tornem al nostre autor. Mauss en un altre article ${ }^{14}$, situant-nos en l'antiga Tràcia, al Nord de l'antiga Grècia i basant-se en els documents escrits de Xenofont, escriu, o més ben dit cita textualment: «...jo els faré els meus germans i els meus convidats i els meus associats en tot allò que podem conquerir. I a tu Xenofont et donaré la meva filla, i si tu tens una filla, jo te la compraré a la manera tràcia i li donaré per residència Bisante, que és el més bell dels territoris marítims... ${ }^{15}$ ¿No és aquest un sistema d'afermar aliances? El mateix Mauss ho explica: «En general, es tracta no d'individus, sinó de col-lectivitats, clans i grans famílies que es comprometen mútuament, molt sovint en forma d'aliança perpètua, en particular a propòsit del matrimoni, a una aliança en el sentit total del conceptes 16 .

Ês evident, doncs, que dins la legalitat, l'esponsalici en tant que document escrit va associat a un contracte de dret privat, $\mathrm{i}$ que dins la moral de l'època està immers en el pes específic de l'Església. I això és un fet innegable. Però tot allò que el document no diu, formalment parlant, és important tenir-ho en compte. Aquí és on s'estableix la unió de les dues disciplines: Antropologia i Història, i al mateix temps el seu límit. Si es distingeixen, en realitat, per l'elecció de perspectives complementàries,

12 Op. cit., M. Mauss: Don, contradon y intercambio.

13 J.E. RUIz.DOMÉNEC: «Système de Parenté et theorie de l'alliance dans la socićté catalane (env. 1000 - cnv, 1240). Revue Historique, 1979.

14 Op. cit., M. Mauss: Sociedad... Una forma antigua de contrato entre los Iracios (1921).

is Xenofont ens proporciona el document principal sobre la induptable institució sumptuària de contracte entre els tracis. (Anábasis, VII).

16 Op. cit., M. Mauss: Sociedad... Una forma antigua... p. 33. 
s'uneixen en un sol fi. La Història organitzarà les seves dades en relació amb les expressions conscients de la vida social, i l'Etnologia en relació amb les inconscients ${ }^{17}$. Això no significa que l'Etnologia sigui indiferent al procés històric, però cercarà a través d'un conscient, que no ignora, un sector cada vegada més gran d'inconscient cap al qual es dirigeix ${ }^{18}$. L'historiador, el medievalista, contràriament, en no tenir a l'abast aquestes perspectives, necessita fer aquest pas envers les aportacions de l'Antropologia per poder anar més lluny.

I donades aquestes premisses, passarem a l'anàlisi dels esponsalicis en qüestió. He establert, en el curs d'aquest treball, una sèrie d'interrogants que em semblen importants, però de fet el punt fonamental d'aquest estudi està projectat en el que representa l'intercanvi dels béns esponsalicis, el principi de l'aliança, el pacte entre dos llinatges, i la continuïtat d'aquesta unió -matrimonial, indissoluble - en el marc de l'estructura familiar. A partir d'aquí analitzarem la documentació:

El primer document ${ }^{19}$ és la donació que fa Ermessendis, uxor que fui Berengarii de Roveria et mater Raimundi de Roveria al Temple del seu sponsalicium, quod fecit michi condam reverendus vir meus Berengarius qui nunc est frater et miles deo gratias ipsius bone milicie. El patrimoni d'aquest esponsalici el té in Roveria et in termino eius et in Rovigone et in termino eius... De fet aquest document permet de veure el retorn de l'esponsalici que va fer Berenguer a Ermessendis quan es varen casar. El fill baró hi és, ella és la mare de Raimon de Roveria i així ho deixa escrit: la continuiitat del llinatge ja està assegurada. Berenguer era frater et miles del Temple. Raimon, el seu fill, també és frater et miles del Temple. L'esponsalici retorna al seu origen. Retorna una altra vegada al llinatge del pare, del seu marit. L'esponsalici ja ha acomplert la seva funció d'aliança i de lligam entre Berenguer i Ermessendis en tant que individualitats, i de llurs llinatges. El document conclou omnia bec dono... in manu Petri de Roveria (germà de Berenguer?) magister eius dei Milicie.

Veurem ara l'esponsalici que fa Pere a Ermengards ${ }^{20}$. Cita que

17 M. Gaborian i d'altres: Estructuralismo e Historia, Buenos Aires 1969, pp. 114-115.

18 Op. cit., M. GABORIAN: Estructuralismo... p. 115

19 A.C.A. Ramon Berenguer IV, n. 169, any 1144.

20 A.C.A. Ramon Berenguer IV, $n$ ? 274, any 1154. 
quan fa la donació encara s'han de casar, l'anomena sponse mee. La cessió és: «... et facio sponsalicio medietate ex mea honore que ego abeo et teneo in cunctis locis in parroechia Sancti Quirici cum terminis et affrontationibus..... I les clàusules hi són: a... remaneat tibi ipsa medietate predicta ab integra potentissime in diebus vite tue... post obitum tuum si abemus prolem legitimi de nos ambi creati remaneat illi sin autem ad propinquis remaneatur....v. Ermengards tindrà el seu esponsalici per tota la vida, perō és el seu marit el que estableix a qui ha de passar la seva donació esponsalícia. Ella n'és l'usufructuària, però no la propietària. Aquesta emeitat» del patrimoni cedit a Ermengards consolida una aliança, però el retorn al llinatge originari s'estableix en la donació. Els fills hi han de ser per tal de gaudir d'aquests béns, però si no, els propinquis ${ }^{21}$ seran els hereus.

Potser el document esponsalici més important, centrat en la meitat del període estudiat, és el que fa Bremundus a Berengue$\mathrm{ra}$, que la vol haver com a legalem uxorem ${ }^{22}$. La donació és prou important: $\kappa . .$. dono tibi in sponsalicio totam compram quam habui de villero de Sancto Mamerta de illo onore qui fuit Guillelmi de Rochafort. Scilicet medietatem castri de Rochafort cum toto onore .... in super totum quod habeo vel habere debeo in villa de Custoga.... Les clàusules són semblants a les del document anterior: "... Si autem me mortuo te non habente prole ex me generata et tu supervixeris haduc totum supra dictum honorem... post mortem vero tuam ad propinquos meos revertaturn. Es clar que el retorn de la donació será als propinquos meos, és a dir al llinatge patern. Si hi ha fills, serà per a ells. Igualment ho demostra l'esponsalici fet per Ramon de Dosrius a la seva muller Saurine ${ }^{23}$ on la clàusula és la següent: «... et si me mori contigerit absque liberis legitimis ad obitum tuum revertatur propinquis meis». I el darrer de Ramon de Carranchano fet a Guilelma qui spero habere per coniuge ${ }^{24}$ totum meum mansum de Charrancho mito tibi in sponsalicio. Per concloure dient e... et si infantes vero defuerint post bobitum vero tuum remaneat meis propinquisw.

21 Propinquis: parents pròxims del llinatge patern.

22 A.C.A. Alfons I, n? 269, any 1166.

23 A.C.A. Alfons I, n: 68, any 1169.

24 A.C.A. Pere I, n: 12, any 1193. 
Si s'intercanvien en una festa esponsalicia els béns esponsalicis i es plasmen, en un document escrit de dret privat, a la Catalunya del segle XII, les donacions i les promeses; donacions econòmiques i promeses matrimonials; aliances de llinatges i esperances de fills continuadors i hereus del patrimoni, no podem denominar l'esponsalici - l'acte - un efet social totals? 


\section{APÈNDIX DOCUMENTAL}

\section{A.C.A. Ramon Berenguer IV n: 169}

Sit notum cunctis tam presentibus quam futuris quod ego Ermessen. dis uxor que fui Berengarii de Roveria et mater Raimundi de Roveria bono animo et bona voluntate per remissione peccatorum meorum et filii mei supradicti atque etiam omnium parentum meorum dono dimitto atque solvo Domino Deo et Milicie Templi Iherosolemitani Salomonis et fratibus ibidem summo regi militantibus presentibus et futuris omnem sponsalicium mei vocem et donum quod sponsalicium fecit michi condam reverendus vir meus Berengarius qui nunc est frater et miles deo gratias ipsius bone milicie. Praeter sicut superius scriptureita dono omne meum sponsalicium quod habeo in Roveria et in termino eius et in Rovigone et in termino eius deo et militibus Templi Salomonis sicut melius dici vel intelligi potest ad voluntatem suam. Omnia hec dono in quocumque loco habeo vel habere debeo in manu Petri de Roveria magister eius dei Milicie. In presencia fratrum videlicet Petri de Arczaz. Raimundi Bernardi.

Actum est hoc viij. kalendas lanuarii. Anno ab incarnacione Dominica M.C.XL.iiij.

Sig+num Ermessendis. Que hoc dono firmo et testes firmare rogo.

Sig+num Raimundi presbiteri. Sig+num Berengarii de Teracia. Sig+num Petri Raimundi presbiteri. Sig+num Marcora. Sig+num Dalmacii.

Sig+num Geraldi Barchinonensis qui hoc scripsit die et anno quo supra.

\section{A. C.A. R. Berenguer IV $n: 274$}

In nomine Domine ego Petrus qui vocant Juliol per hanc kartam dona. tionis spontanea voluntate dono tibi sponse mee Ermengards et facio sponsalicio medietate ex mea honore que ego abeo et teneo et abere debeo in cunctis locis in parroechia Sancti Quirici cum terminis et affrontationibus cum exiis atque regresiis suis. Tali modo sic dono tibi medietate predicta ab integra ex mea honore ut teneamus et abeamus in simul ambi dum vivamus et post meum díscessum si obiero ego Petri prescripti ante te uxori mee predicte remaneat tibi ipsa medietate predicta ab integra potentissime in diebus vite tue sine contrarietate ullius homo vel fe- 
mine cum prole et sine prole cum marito et absque marito post obitum tuum si abemus prolem legitimi de nos ambi creati remaneat illi sin autern ad propinquis remaneatur. Si quis hoc fringere voluerit in duplo componat. Actum est hoc .viij. kalendas septembris. Anno .xviij. Regni regis Ludovici iuniori.

Sigtnum Perri qui hoc donum vel sponsalicium facio et firmo er firmarique rogo. Sigtnum Berengarii fratris eius qui hoc laudo et firmo Sigtnum Raimundi Faber. Sig+num Guillelmi de Combas. Sig+num Ermesndi domina mater eius.

Guillelmus presbiter qui hoc scripsit di et anno quo + supra.

\section{A.C.A. Alfons I n: 209}

In nomine Domini Nostri Ihesu Christi anno ab incarnatione eiusdem M.C.Lx.vj. mense augusti .v. kalendas septembris feria .j.

Ego Bremundus quia te Berenguariam volo habere legalem uxorem et exte legalem prolem suscipere cupio me ipsum trado tibi in legalem virum. Et sit notum quod dono tibi in sponsalicio totam compram quam habui de villero de Sancto Mamerta de illo onore qui fuit Guillelmi de Rochafort. Scilicet medietatem castri de Rochafort cum toto onore illo quem comparavi cum castro predicto in super totum quod habeo vel habere debeo in villa de Custoga et eius terminis quod fuit de hac eadem compram prenominata. Tali vero ratione ut nos hoc totum onorem habeamus et teneamus dum vixerimus et in mortem nostram infantes nostri habeant si habuerimus. Si autem me mortuo te non habente prole ex me generata et tu super vixeris haduc totum supradictum onorem. habeas et teneas in omni vita tua. Post mortem vero tuam ad propinquos meos revertatur. Sit quoque notum quod ego a te habui pro tua ereditate mille solidos melgoriensium quos ego laudo tibi in predicto onote quem onorem tamdiu proximi tui vel ille cui tu dimiseris teneant donec proximi mei istos predictos mille solidos persolvant. In super dono tibi uxori mee Berengarie de omnibus que ad hodie in antea tecum adquisiero tuam plenam porcionem secundum consetudinem narbonensis parte. Sit autem memoriale quod die huius scripcionis marca argenti valet. L. solidos melgores. Facta carta anno et die quo supra regnante Lodovico rege in Francia.

Sig+num Bremundi qui hanc cartam fieri feci firmari qui rogavi. Sigtnum Bedocii. Sig+num Berengarii fratrum mei Bremundi de Segano. Sig+num Ermengarde vicecomitisse de Narbona. Sig+num Aimerici de Narbona. Sig+num Petri abbatis Sancti Pauli. Sigtnum Hermengaudi de Leocata. Sig+num Hermengaudi de Vernet. Sig+num Raimundi de 
Calis. Sig +num Petri Raimundi vicarii de Narbona. Sig +num Raimundi de Durban. Sig+num Jordani de Mitationibus. Sig+num Petri de Cacio Castello. Sig+num Petri Bernardi sacriste Sancti lusti et Pastoris.

Sig+num Bremundi percentoris Sancti lusti qui hanc cartam rogatus scripsit.

\section{A.C.A. Alfons I n: 68}

In nomine Domini ego Raimundus de duobus rivis consilio et voluntate patris mei Raimundi et aliorum amicorum meorum dono tibi Saurine uxori mee in sponsalicium totum illum honorem tam alodium quam fevum quem habemus ego et pater meus in parrohia Sancte Marie de Mesleo et in parrochia Sancti Martini de Cotibus et in parrochia Sancte Cecilie de Voldrega. Tali pacto dono tibi Saurine uxori mee in sponsalicium totum illum honorem tam alodium quam fevum quem ego et pater meus habemus et habere debemus in predictis partochiis et homines per nos cum nostro manso de Podio qui est in parrochia Sancti Petri de Torilione. Ut tu hoc totum teneas potenter cum marito et sine marito et cum infante et (sine) absque infante in omni vita tua et si infantes habuerimus ex me in te genitos remaneat eis et progeniei eorum post obitum meum et tuum et si me mori contingerit absque liberis legitimis ad obitum tuum revertatur propinquis meis. Et si forte predictus pater meus Raimundus obierit antea ego et tu habeas tu totum illum meum honorem tam alodium quam fevum quem habeo et habere debeo et homines et homines per me infra terminos de Torilione in sponsalicium et meum estai de Torilione et ego recuperem predictum sponsalicium excepto manso de Podio ad meam voluntatem. Et si forte te Saurinam infirmari contingerir sit tibi licitum facere testamentum ex illo avere quem in te accipio et si forte absque liberis legitimis de me in te procreatis intestata obieris teneant propinqui tui hunc tuum sponsalicium donec ego aut propinqui mei reddidamus eis mille solidos monete Barchinone. Si quis hoc dirumpere voluerit agere non valeat set in duplum componat et in super firmum permaneat.

Quod est factum .vij, kalendas iunii. Anno .xxxij. regni regis Lodovici iunioris.

Sig+num Raimundi de Duobus rivis. Qui hoc facio et firmo firmarique rogo. Sigtnum Raimundi patris eius. Sig+num Bernardi de Mesteo. Sig+num Guillelmi de Moncada. Sig+num Guillelmi Bernardi de Mesteo. Sig+num Godalli de Cosul. Sig+num Guillelmi filii eius. Sig+num Bernardi de Gurbo. Sig+num Berengarii de Bello Podio. Sig+num Poncio de Torilione. Sig+num Petri de Noguer. Bernardi presbiter. 
Raimundus sacerdos qui hoc scripsit cum litteris suprascriptis in ,vij. linea die et anno quo + supra.

Hoc est translatum sumptum fideliter a quodam instrumento cuius series talis est. Notum sit cunctis quod ego Raimundus de Carranchano cum assensu et voluntate matris mee dono tibi Guillelme qui spero habere per coniuge totum meum mansum de charrancho mito tibi in sponsalicium sicut dominus milicie Templi Salomonis nobis dederint in nostra precharia sicut mito tibi in sponsalicium cum introitibus et exibus suis totum ab integrum sicut melius dicit vel intelligi potest. Tali modo ut habeas et teneas mecum omnibus diebus vite tue et post hobitum vero tuum si habueris infantem procreatum de me in te abeant illos et si infantes vero defuerint post hobitum vero tuum remaneat meis propinquis salvo iure dominorum. Et si quicumque de meis propinquis voluerit tradere de tua potestate suum sponsalicium facere non posit. Donec habeat tibi $[. .$.$] persolutos .CCCCL. solidos denariorum bone monete$ Barchinone valentis marcha argenti .xL.iiij. ${ }^{\circ}$. Si quis hoc fregerit [...] in duplo componat et post hec carta firma maneat omni tempore. Actum est hoc .iiij. kalendas septembris. Anno Domini [...] Sig num Raimundi de Carranchano qui hoc donum facio et firmo firmarique rogo. Sig+num [...] matris mee. Sig +num Guillelmi de [...] precentor milicie palatii. Sig+num frater Raimundi. Sig+num [...] de Galifa. Sig+num Bernardi de Mascharens. Sig+num Berengarii Compani.

Sig+num Arnaldi lebite qui hoc scripsi cum literis rasis et esmendatis in linea viiii. die et anno quo supra. 